\title{
NO-Releasing Nanoparticles Ameliorate Detrusor Overactivity in Transgenic Sickle Cell Mice via Restored NO/ROCK Signaling
}

\author{
Serkan Karakus, Biljana Musicki, Mahantesh S. Navati, Joel M. Friedman, \\ Kelvin P. Davies, and Arthur L. Burnett \\ The James Buchanan Brady Urological Institute and Department of Urology, The Johns Hopkins, University School of Medicine, \\ Baltimore, Maryland (S.K., B.M., A.L.B.); and Departments of Physiology and Biophysics (M.S.N., J.M.F., K.P.D.) and Urology \\ (K.P.D.), Albert Einstein College of Medicine, New York, New York
}

Received December 18, 2019; accepted March 2, 2020

\begin{abstract}
Sickle cell disease $(S C D)$ is associated with overactive bladder (OAB). Detrusor overactivity, a component of $\mathrm{OAB}$, is present in an SCD mouse, but the molecular mechanisms for this condition are not well-defined. We hypothesize that nitric oxide (NO)/ ras homolog gene family (Rho) A/Rho-associated kinase (ROCK) dysregulation is a mechanism for detrusor overactivity and that NO-releasing nanoparticles (NO-nps), a novel NO delivery system, may serve to treat this condition. Male adult SCD transgenic, combined endothelial NO synthases (eNOSs) and neuronal NOS (nNOS) gene-deficient $\left(\mathrm{dNOS}^{-1-}\right)$, and wild-type (WT) mice were used. Empty nanoparticle or NO-np was injected into the bladder, followed by cystometric studies. The expression levels of phosphorylated eNOS (Ser-1177), protein kinase B (Akt) (Ser-473), nNOS (Ser-1412), and myosin phosphatase target subunit 1 (MYPT1) (Thr696) were assessed in the bladder. SCD and $\mathrm{dNOS}^{-1-}$ mice had a greater $(P<0.05)$ number of voiding and nonvoiding contractions compared with WT mice, and they were normalized by NO-np treatment. eNOS (Ser-1177) and AKT (Ser-473) phosphorylation were decreased $(P<0.05)$ in the bladder of SCD compared with WT
\end{abstract}

mice and reversed by NO-np. Phosphorylated MYPT1, a marker of the RhoA/ROCK pathway, was increased $(P<0.05)$ in the bladder of SCD mice compared with WT and reversed by NO-np. nNOS phosphorylation on positive (Ser-1412) regulatory site was decreased $(P<0.05)$ in the bladder of SCD mice compared with WT and was not affected by NO-np. NO-nps did not affect any of the measured parameters in WT mice. In conclusion, dysregulation of $\mathrm{NO}$ and RhoA/ROCK pathways is associated with detrusor overactivity in SCD mice; NO-np reverses these molecular derangements in the bladder and decreases detrusor overactivity.

\section{SIGNIFICANCE STATEMENT}

Voiding abnormalities commonly affect patients with sickle cell disease (SCD) but are problematic to treat. Clarification of the science for this condition in an animal model of SCD may lead to improved interventions for it. Our findings suggest that novel topical delivery of a vasorelaxant agent nitric oxide into the bladder of these mice corrects overactive bladder by improving deranged bladder physiology regulatory signaling.

\section{Introduction}

The urinary bladder is a unique organ that has a dual function; it relaxes while filling with urine and contracts to expel urine during micturition. Normal bladder function requires a coordinated and complex interaction between the brain, spinal cord, and lower urinary tract (LUT). Various stimuli induce release of chemical mediators from the urothelium. Disturbances between the nervous system and the urothelial cells may lead to sensory symptoms, such as urinary frequency and urgency (Abrams et al., 2017). Neuronal nitric oxide (NO) is a urothelial signaling molecule that increases the permeability of urothelium and reduces the activity of detrusor smooth muscle (DSM) via inhibiting the activity of

The study was supported by grant from the National Institutes of Health, [NIH/NIDDK Grant R56DK114095 to A.L.B.].

No conflicts of interest, financial or otherwise, are declared by the authors. https://doi.org/10.1124/jpet.119.264697. bladder afferent nerves (Lavelle et al., 2000; Pandita et al., 2000; Parsons, 2007). However, whether NO exerts direct action on DSM is unclear. Intravesical NO scavenging and systemic NO synthase (NOS) inhibition result in detrusor overactivity in mice and rats, whereas NO donors reduce detrusor contraction frequency (Burnett et al., 1997; Ozawa et al., 1999; Pandita et al., 2000; Persson et al., 2000; Kamiyama et al., 2008; Mónica et al., 2008; Caremel et al., 2010). On the other hand, spontaneous activity in the DSM may be enhanced by NO through NO/cGMP-independent mechanisms (Yanai et al., 2008; Meng et al., 2012). Furthermore, a complex response (contraction, relaxation, or biphasic) of DSM to NO donors and cGMP analogs has been shown in isolated human and animal detrusor strips (Moon, 2002). The understanding of NO's role in the physiology and pathophysiology of the LUT remains unclear and warrants further studies.

Detrusor overactivity represents a major underlying pathophysiology of overactive bladder (OAB) syndrome and is

ABBREVIATIONS: Akt, protein kinase B; $\mathrm{dNOS}^{-1-}$, combined eNOS and nNOS gene-deficient; DSM, detrusor smooth muscle; empty-np, empty nanoparticle; eNOS, endothelial NOS; LUT, lower urinary tract; MYPT1, myosin phosphatase target subunit 1; nNOS, neuronal NOS; NO, nitric oxide; NO-np, NO-releasing nanoparticles; NOS, NO synthase; NVC, nonvoiding contraction; OAB, overactive bladder; P-, phosphorylated; Rho, ras homolog gene family; ROCK, Rho-associated kinase; SCD, sickle cell disease; VC, voiding contraction; WT, wild type. 
defined by urodynamic criteria as involuntary detrusor contractions during the filling phase. The prevalence of OAB has been estimated to be between $16.5 \%$ and $23.2 \%$ in the general population of the United States and as high as $40 \%$ in patients with sickle cell disease (SCD) (Portocarrero et al., 2012; Anele et al., 2016; Powell et al., 2018). SCD is an inherited disorder characterized by an abnormal structure of one of the globin chains of the $\mathrm{Hb}$ molecules, resulting in multiple clinical presentations. The chronic state of reduced NO bioavailability plays a significant role in the pathogenesis of SCD, and prior studies have implicated dysregulation of the NO-signaling pathway in genitourinary dysfunctions in SCD, such as priapism and OAB (Silva et al., 2016; Karakuset al., 2019; Musicki et al., 2019).

In our previous study, we showed that chronically dysregulated NO signaling in LUT contributes to detrusor overactivity and urethral dysfunction in SCD, whereas long-term dietary inorganic nitrate supplementation corrected the OAB phenotype by reversing NO deficiency (Musicki et al., 2019). In this study, we evaluated the effect of NO deficiency and NO replacement with a novel NO therapy, NO-releasing nanoparticles (NO-nps), on voiding function and molecular signaling in the bladder using two mouse models of NO deficiency: transgenic SCD mice and combined endothelial NOS (eNOS) and neuronal NOS (nNOS) gene-deficient $\left(\mathrm{dNOS}^{-/-}\right)$mice.

\section{Material and Methods}

Animals. Male 3.3-6-month-old, age-matched SCD transgenic, $\mathrm{dNOS}^{-/-}$, and wild-type (WT) mice were used for the following evaluations: cystometry (3.6-6-month-old) and Western blot analysis (3.3-5.6-month-old). Transgenic SCD mouse breeding pairs (strain 3342) were obtained from Jackson Laboratory (Bar Harbor, ME) and generated by knockout of mouse $\alpha$ and $\beta$ globins and insertion of a single transgene that expresses human $\alpha$ and $\beta$ sickle globin (Pászty et al., 1997). SCD mice were bred in-house. Genotyping was performed by Transnetyx, Inc. (Cordova, TN). Adult male $\mathrm{dNOS}^{-} /^{-}$mice, originally obtained from Dr. Paul L. Huang and bred in-house were used as a secondary and confirmatory model of NO deficiency (Huang et al., 1993). WT C57BL/6 male mice (The Jackson Laboratory) were used as controls representing the strains for the predominant genetic background of the transgenic SCD and $\mathrm{dNOS}^{-1-}$ mice. Mice were kept in a germ-free animal facility with a 12/12-hour day/night cycle. All animal studies were conducted in accordance with ethical standards of the Johns Hopkins University School of Medicine Guidelines for the Care and Use of Animals.

Nanoparticles Preparation. The synthesis of NO-np and empty$\mathrm{np}$ was recently reported (Friedman et al., 2008). A fine powder comprised of $10 \mathrm{~nm}$ empty nanoparticles or $10 \mathrm{nM}$ NO nanoparticles was mixed with saline $(0.9$ and $5 \mathrm{mg} / \mathrm{ml}$, respectively) and afterward with polyethylene glycol solution ( 12.5 and $10 \mu \mathrm{l} / \mathrm{mg}$, respectively) just before use (Han et al., 2010; Tar et al., 2014). Once exposed to an aqueous environment, the hydrogel properties of the composite allow opening of the water channels inside the particles, facilitating the release of $\mathrm{NO}$, which lasts up to several hours.

Cystometry. Thirty-seven mice ( $n=4-9$ /group) underwent cystometry. Intraperitoneal injection of urethane $(1.8 \mathrm{mg} / \mathrm{kg})$ was used for anesthesia. Once the bladder was exposed via lower abdominal laparotomy, mice were allowed to stabilize for 20-30 minutes before NO-np injections. After emptying the bladder, $50 \mu$ l of empty-np or NO-np dispersion was injected by a 31-gauge needle into the bladder. A 25-gauge butterfly needle that was connected to a two-way tap was then inserted into the bladder dome through the same needle hole after 10 minutes. One of these ports was connected to a pressure transducer, and the other port was connected to an infusion pump.
Cystometry was continuously performed for 30 minutes by infusing saline in the bladder at $10 \mu \mathrm{l} / \mathrm{min}$. The parameters assessed were peak pressure (maximum pressure observed during a micturition cycle), bladder capacity (maximum volume infused into the emptied bladder before urine expulsion, assessed at the end of the cystometry), compliance (change in volume/change in baseline and threshold pressures before first voiding contraction occurred), and the frequency of voiding contractions (VCs) and nonvoiding contractions (NVCs). VCs were defined as rises in intravesical pressure that exceeded $4 \mathrm{~mm}$ $\mathrm{Hg}$ and were associated with a saline expulsion from the urethra (micturition). The contractions that were not accompanied by micturition were defined as NVCs (Leiria et al., 2013; Karakuset al., 2019). Cystometry data were recorded using the DI-190 system (Dataq Instruments, Akron, $\mathrm{OH}$ ).

Western Blot Analysis. Bladder samples were collected 40 minutes after intravesical injection of $50 \mu \mathrm{l}$ of empty-np or $50 \mu \mathrm{l}$ of NO-np from a separate group of anesthetized mice $(n=24 ; 4-10$ mice/group) that did not undergo cystometric evaluation. The tissue was snap-frozen in liquid nitrogen and stored at $-80^{\circ} \mathrm{C}$ until processed for Western blot analyses. The bladder was homogenized as previously described (Karakuset al., 2017). Protein concentration was determined using the bicinchoninic acid method. Homogenates $(90 \mu \mathrm{g})$ were resolved on $4 \%-20 \%$ Tris gels and transferred to polyvinylidene difluoride membranes. Membranes were blocked for 1 hour at room temperature in PBS ( $\mathrm{pH} 7.4$ ) containing $0.1 \%$ Tween-20 and $5 \%$ nonfat dry milk and then probed overnight at $4^{\circ} \mathrm{C}$ in PBS containing $0.1 \%$ Tween-20 and 3\% nonfat dry milk with the following antibodies: rabbit anti-phospho- (P-) eNOS (Ser-1177) (1:450 dilution, catalog 9571S; Cell Signaling Technology, Beverly, MA), rabbit anti-P-nNOS (Ser-1412) (1:7000 dilution, kindly provided by Dr. Solomon Snyder, Johns Hopkins University, Baltimore, MD) (Hurt et al., 2012), rabbit anti-P-myosin phosphatase target subunit 1 (MYPT1) (Thr-696) (dilution 1:1000, catalog 5163; Cell Signaling Technology), and rabbit anti-P-Akt (Ser-473) (dilution 1:1000, catalog 9271; Cell Signaling Technology). Signals were standardized to eNOS (monoclonal anti-eNOS at 1:1000 dilution, catalog 610296; BD Transduction, Laboratories, San Diego, CA), nNOS (polyclonal anti-nNOS at 1:9000 dilution, kindly provided by Dr. Solomon Snyder), MYPT1 (polyclonal anti-MYPT1 at 1:1000 dilution, catalog sc-25618; Santa Cruz Biotechnology, Dallas, TX), or Akt (polyclonal anti-Akt at 1:1000 dilution, catalog 9272; Cell Signaling Technology). Bands were detected by horseradish peroxidase-conjugated anti-mouse or antirabbit secondary antibodies (1:7000 dilutions, catalog NA931V and NA934V; GE Healthcare) and quantified using NIH Image 1.29 software (National Institutes of Health). Results were expressed relative to those of WT mice treated with empty-np.

Statistical Analysis. Data are expressed as mean \pm S.E.M. Statistical analyses were performed using one-way ANOVA followed by post hoc analysis using the Tukey-Kramer test (Prism v.5; GraphPad Software, San Diego, CA). For comparison of Western blot data between WT mice treated with empty-np and each treatment group, a modified $t$ test was used to compare the experimental groups with the normalized control ratio. A value of $P<0.05$ was considered statistically significant.

\section{Results}

NO-np Improved Abnormal Cystometric Voiding and Nonvoiding Frequencies in SCD and $\mathrm{dNOS}^{-/-}$Mice. Cystometrogram tracings showed that SCD and $\mathrm{dNOS}^{-/-}$ mice had a greater number of VCs and NVCs and low compliance compared with WT mice (Fig. 1). Both voiding (Fig. 2A) and nonvoiding (Fig. 2B) frequencies were increased $(P<0.05)$ in $\mathrm{dNOS}^{-1-}$ and SCD mice compared with those of WT mice. Bladder compliance was decreased $(P<0.05)$ in $\mathrm{dNOS}^{-1-}$ and SCD mice compared with that of WT mice (Fig. 2C). Peak pressure was decreased $(P<0.05)$ in $\mathrm{dNOS}^{-/-}$ 

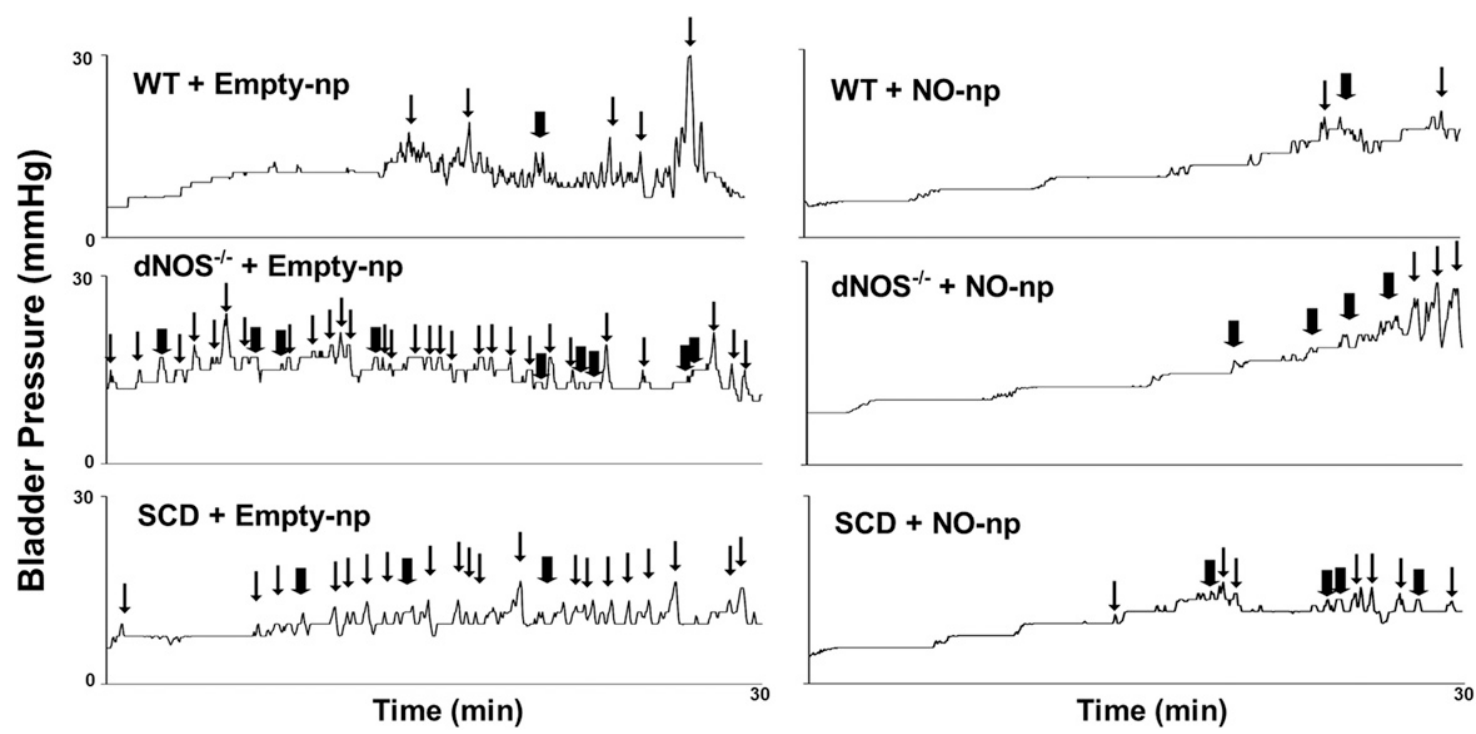

Fig. 1. Representative cystometrogram traces in WT + Empty-np, WT + NO-np, dNOS ${ }^{-1-}+$ Empty-np, dNOS ${ }^{-1-}+$ NO-np, SCD + Empty-np, and SCD + NO-np. dayNOS ${ }^{-1-}$ and SCD mice exhibit unstable bladder contractions. Thick arrows indicate nonvoiding contractions, thin arrows indicate voiding contractions.

mice and slightly decreased in SCD mice compared with that of WT mice $(P=0.0565)$ (Fig. 2D). There were no significant differences in bladder capacity among groups (Fig. 2E). Treatment of SCD and $\mathrm{dNOS}^{-/-}$mice with NO-np decreased $(P<$ $0.05)$ the number of VCs and NVCs to levels measured in WT mice (Figs. 1 and 2). NO-np treatment did not affect bladder compliance, peak pressure, and capacity in either SCD or $\mathrm{dNOS}^{-1-}$ mice. NO-np treatment did not affect cystometric parameters in the bladder of WT mice.

Protein expressions of P-eNOS (Ser-1177), P-Akt (Ser-473), and P-nNOS (Ser-1412) were decreased in the SCD mouse bladder; protein expressions of P-eNOS (Ser-1177) and P-Akt (Ser-473) were normalized by NO-np treatment. Activated (phosphorylated) forms of eNOS (Ser-1177) and Akt (Ser-473) were decreased $(P<0.05)$ in the SCD mouse bladder compared with those of WT mice and were increased $(P<$ 0.05 ) by NO-np treatment to levels similar to those measured in WT mice (Fig. 3, A and B). Activated, phosphorylated nNOS (Ser-1412) was also decreased $(P<0.05)$ in the SCD mouse bladder compared with that of WT mice and was not affected by NO-np treatment. (Fig. 3C). NO-np administration did not affect phosphorylated eNOS, nNOS, or Akt in the WT mouse bladder.

Protein Expression of P-MYPT1 Was Increased in the SCD Mouse Bladder and Normalized by NO-np Treatment. The ratio of P-MYPT1 (Thr-696) to total MYPT1,
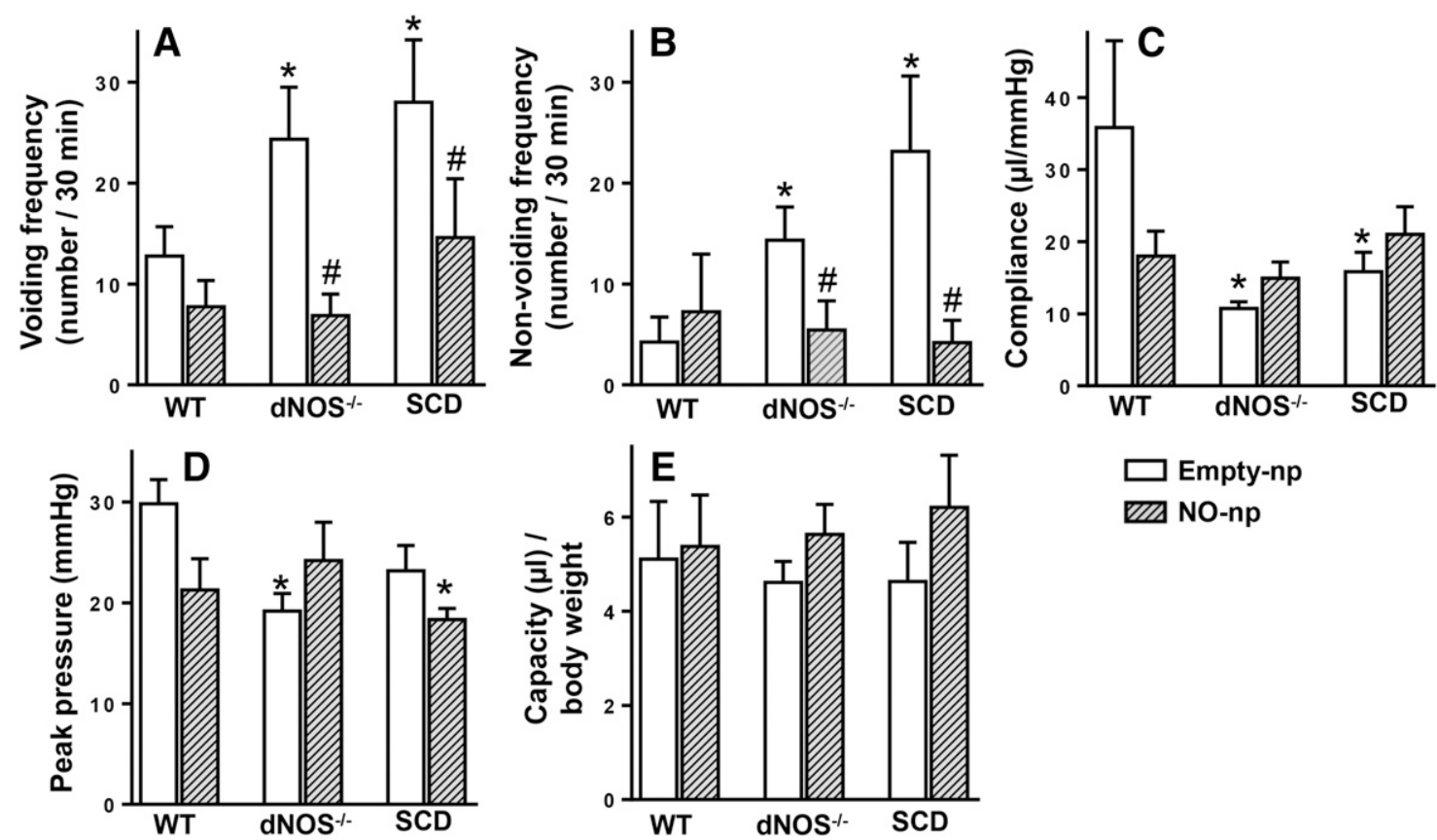

Fig. 2. Effect of NO-np on cystometric parameters [voiding frequency (A), nonvoiding frequency (B), compliance (C), peak pressure (D), and capacity (E)] in WT, $\mathrm{dNOS}^{-/-}$, and SCD mice. Each bar represents the mean \pm S.E.M. for four to nine mice in each group. ${ }^{*} P<0.05$ vs. WT; ${ }^{\#} P<0.05$ vs. Empty-np. 
reflecting RhoA/Rho-associated kinase (ROCK) activity, was increased $(P<0.05)$ in the SCD mouse bladder compared with that of WT mice. NO-np treatment decreased $(P<0.05)$ protein expression of P-MYPT1 in the SCD mouse bladder to levels similar to those measured in WT mice (Fig. 3D). NO-np administration did not affect phosphorylated MYPT1 in the WT mouse bladder.

\section{Discussion}

This study demonstrates that intravesical application of NO-np, a new NO delivery system, reversed OAB phenotype in an SCD mouse model of chronic NO deficiency, as documented by decreased voiding and nonvoiding contraction frequencies. This effect of NO-np is associated with normalization of molecular signaling in the bladder involving Akt/ NOS relaxing and RhoA/ROCK contractile signaling pathways. The lack of NO-np effect on NO signaling in WT animals is in line with previous findings in the penis of healthy adult rats and healthy adult men with other NO-stimulating compounds, such as phosphodiesterase type 5 inhibitors (Mondaini et al., 2003; Musicki et al., 2005). Although NO is understood to be a weak relaxing agent in the bladder under physiologic conditions, its effect may actually be more evident under pathologic conditions.

In this study, we confirmed our recent finding of $\mathrm{OAB}$ phenotype in an SCD mouse model (Karakuset al., 2019; Musicki et al., 2019), which is in line with clinical observations that pediatric and adult populations of patients with SCD exhibit urinary symptoms, such as OAB and nocturnal enuresis (Anele et al., 2016). We obtained similar cystometric results using another mouse model of chronic NO depletion, the dNOS knockout mouse, further substantiating the importance of $\mathrm{NO}$ deficiency in the pathogenesis of OAB. Here, we provide an explanation for the derangement in molecular signaling resulting in OAB using a humanized SCD animal model: Activities of both constitutive NOS isoforms are decreased, whereas the RhoA/ROCK contractile pathway is increased in the bladder, both of which contribute to enhanced detrusor overactivity.

The molecular mechanism underlying the reduction in NOS activity in the SCD mouse bladder involves decreased phosphorylation of both constitutive NOS isoforms on positive regulatory sites (Ser-1177 and Ser-1412 on eNOS and nNOS, respectively) and decreased phosphorylation of Akt, an activator of eNOS. These results confirm and extend our recent findings (Musicki et al., 2019).

We show here for the first time that the SCD mouse bladder exhibits increased activity of the RhoA/ROCK contractile pathway. We documented this by demonstrating increased phosphorylation of MYPT1, a surrogate marker of the RhoA/ ROCK pathway activity. MYPT1 is a regulatory subunit of myosin light chain phosphatase, which dephosphorylates the myosin light chain and initiates relaxation of smooth muscle cells at low calcium levels (Gallagher et al., 1997; Grassie et al., 2011). Phosphorylation of MYPT1 on Thr-696 by ROCK inhibits the phosphatase activity of myosin light chain phosphatase and thereby causes smooth muscle contraction. Components of the RhoA/ROCK-signaling pathway are expressed in the detrusor and play important roles in regulating detrusor contraction and tone (Teixeira et al., 2007). Previous studies showed that RhoA/ROCK activity
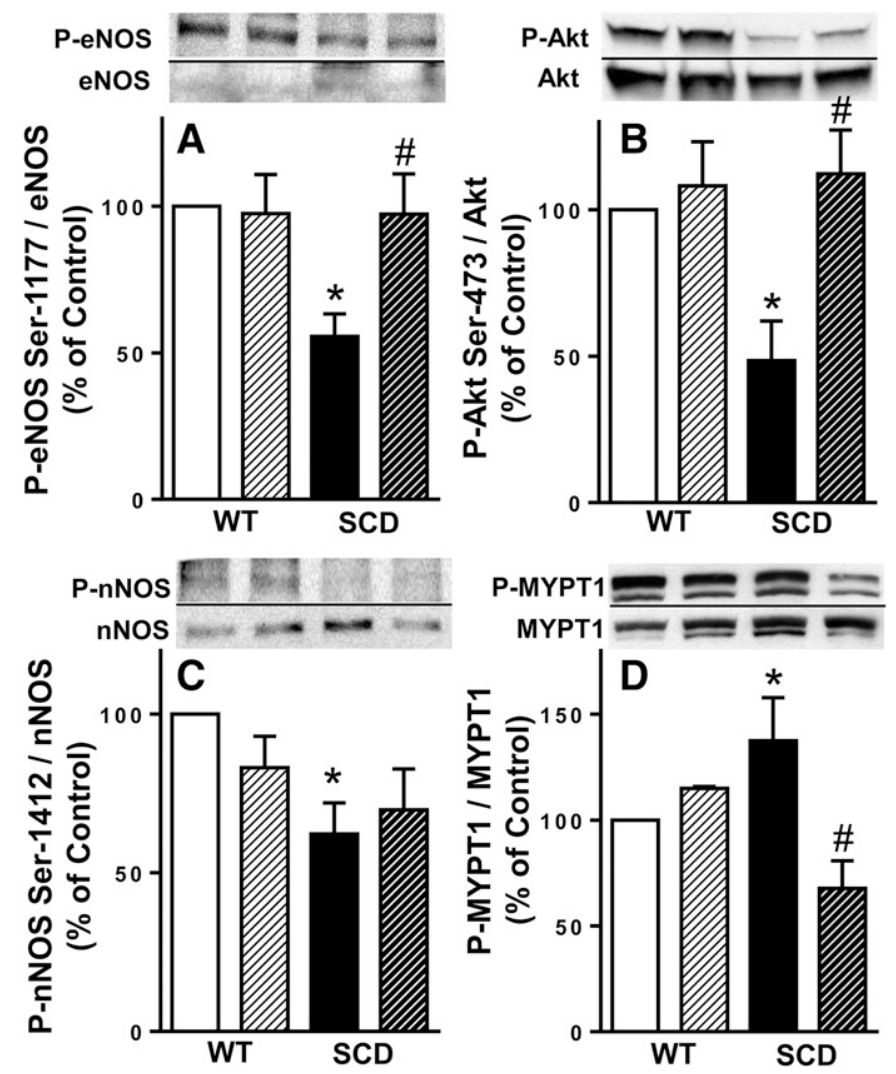

Fig. 3. Upper panels are representative Western immunoblots of P-eNOS (Ser-1177), eNOS, P-Akt (Ser-473), Akt, P-nNOS (Ser-1412), nNOS, P-MYPT1, and MYPT1 in the bladder of WT + Empty-np, WT + NO-np, SCD + Empty-np, and SCD + NO-np. Lower panel represents quantitative analysis of P-eNOS (Ser-1177), eNOS, P-Akt (Ser-473), Akt, P-nNOS (Ser-1412), nNOS, P-MYPT1, and MYPT1 in the same treatment groups. Each bar represents the mean \pm S.E.M. for $4-10$ mice in each group. ${ }^{*} P<0.05$ vs. WT, ${ }^{\#} P<0.05$ vs. Empty-np.

was increased in the OAB bladder in animal models of $S$-nitrosoglutathione reductase deficiency, bladder outlet obstruction, and hypertension (Teixeira et al., 2007; Akakpo et al., 2017; Wróbel et al., 2017). An inverse functional relationship exists between the NO/cGMP/protein kinasez G and RhoA/ROCK-signaling pathways (Laufsand(Laufs and Liao, 1998) . Activation of RhoA/ROCK decreases both eNOS protein expression and Akt-dependent eNOS phosphorylation on Ser-1177 (Ming et al., 2002; Bolz et al., 2003). Conversely, cGMP-dependent protein kinase $\mathrm{G}$ phosphorylates RhoA on Ser-188 and prevents its translocation to the membrane, thus preventing its activation and inhibiting the RhoA/ROCK pathway (Kizub et al., 2010; Cicek et al., 2013). Although the mechanism of increased RhoA/ROCK activity in OAB in SCD is not known, it is plausible that it is induced by chronic low NO bioavailability. Upregulated activity of the RhoA/ROCK pathway in the SCD mouse bladder conceivably increases smooth muscle contractility and contributes to the OAB phenotype.

We next used a novel NO delivery system not described before for intravesical NO delivery to replenish NO bioavailability in the SCD mouse bladder. NO-np is a true NO generator, not an NO-donating compound with potential cytotoxicity, inadequate release capacity, and instability, and allows localized delivery of the gaseous NO. The nanoparticles uniquely facilitate the formation of NO from nitrite 


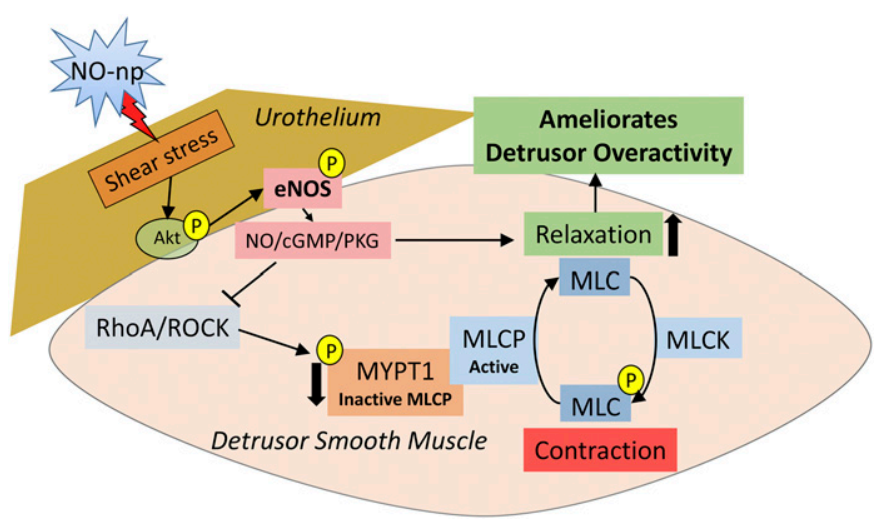

Fig. 4. Proposed mechanism of NO-np action on amelioration of detrusor overactivity in the SCD mouse. The molecular mechanism underlying detrusor overactivity in the SCD mouse involves the reduction in Akt/ eNOS activities (reduced phosphorylation of Akt on Ser-473 and eNOS on Ser-1177) and increased RhoA/ROCK contractile pathway (increased phosphorylation of MYPT1, a regulatory subunit of MLCP, on Thr-696). NO-np treatment increases Akt/eNOS activities and decreases RhoA/ ROCK activity in the SCD mouse bladder by enhancing blood flow and shear forces on the urothelium. Decreased phosphorylation of MYPT1 activates MLCP, which dephosphorylates the MLC and initiates relaxation of smooth muscles. Normalized Akt/eNOS and RhoA/ROCK activities ameliorate detrusor overactivity. MLC, myosin light chain; MLCK, myosin light chain kinase; MLCP, myosin light chain phosphatase.

salt through a stable and potent $\mathrm{NO}$ intermediate, $\mathrm{N}_{2} \mathrm{O}_{3}$. NOnps are stable and produce no cytotoxicity or widespread adverse effects (Tar et al., 2014). Their previous use in the penis, namely for treatment of erectile dysfunction, is associated with aging, diabetes, and cavernous nerve injury (Han et al., 2010; Draganski et al., 2018), indicates their effectiveness in NO delivery. We show here that NO-np treatment reversed detrusor overactivity in both SCD and $\mathrm{dNOS}^{-1-}$ mouse models and, as expected with only short-term treatment, did not affect bladder capacity, peak pressure, or compliance. This effect of NO-np treatment was associated with a reversal of downregulated Akt/eNOS activities and reversal of upregulated ROCK activity in the SCD mouse bladder. We propose that increased local NO by NO-np treatment resulted in enhanced blood flow and shear forces on the urothelium, activated Akt/eNOS (Ser-1177) phosphorylation cascade, and promoted NO release and relaxation of the bladder (Dimmeler et al., 1999; Hurt et al., 2002; Sriram et al., 2016). Increased NO may further downregulate the contractile ROCK pathway activity in the bladder, further improving bladder relaxation (Fig. 4).

The nNOS phosphorylation site 1412 is a target for Akt and protein kinase A in some systems (Förstermann and Sessa, 2012). We found no effect of NO-np on nNOS phosphorylation on Ser-1412, suggesting that the beneficial effects of NO in ameliorating the OAB phenotype in the SCD mouse are apparently not mediated by increased nNOS phosphorylation, at least with short-term NO-np treatment. Although several studies reported higher leak point pressure and bladder capacity and absent urethral relaxation in mice lacking nNOS (Burnett et al., 1997; Sutherland et al., 1997), the role of nNOS activation by phosphorylation in the LUT awaits further evaluation.

There were several possible limitations of our study. First, SCD and $\mathrm{dNOS}^{-/-}$mice likely possess metabolic or nephrological abnormalities as well as hemolysis-related blood flow disturbances that may contribute to voiding dysfunction (Morishita et al., 2005). Additionally, utilization of the transgene or gene-knockout models may have produced effects independent of NO signaling affecting voiding function in these mice. Second, we did not directly quantify NO release into the bladder. A separate study is warranted to elucidate the nanoparticles' penetration profiles in the bladder. Third, further studies are also needed to delineate possible differences in bladder morphology between the WT and SCD mice. Fourth, our study was limited to male SCD mice. Possible sex differences in NO deficiency in $\mathrm{OAB}$ can be further investigated using female SCD mice.

Our results demonstrate that SCD mice exhibit an OAB phenotype due to NOS dysregulation and upregulation of the RhoA/ROCK contractile pathway in the bladder; NO-np normalized detrusor overactivity while reversing these molecular abnormalities in the bladder. Our findings suggest the topical application of NO-np as a novel approach for treatment of OAB by targeting the NO-signaling pathway. Although our studies show short-term effects of NO-np, the potential longterm beneficial effects on $\mathrm{OAB}$ await further investigation.

\section{Authorship Contributions}

Participated in research design: Karakus, Burnett.

Conducted experiments: Karakus.

Contributed new reagents or analytic tools: Navati, Friedman, Davies.

Performed data analysis: Karakus.

Wrote or contributed to the writing of the manuscript: Karakus, Musicki, Burnett.

\section{References}

Abrams P, Cardozo L, Wagg A, and Wein A (2017) in Incontinence 6th Edition. ICIICS, International Continence Society, Bristol, UK.

Akakpo W, Musicki B, and Burnett AL (2017) cAMP-dependent regulation of RhoA/ Rho-kinase attenuates detrusor overactivity in a novel mouse experimental model. BJU Int 120:143-151.

Anele UA, Morrison BF, Reid ME, Madden W, Foster S, and Burnett AL (2016) Overactive bladder in adults with sickle cell disease. Neurourol Urodyn 35: 642-646.

Bolz SS, Vogel L, Sollinger D, Derwand R, de Wit C, Loirand G, and Pohl U (2003) Nitric oxide-induced decrease in calcium sensitivity of resistance arteries is attributable to activation of the myosin light chain phosphatase and antagonized by the RhoA/Rho kinase pathway. Circulation 107:3081-3087.

Burnett AL, Calvin DC, Chamness SL, Liu JX, Nelson RJ, Klein SL, Dawson VL, Dawson TM, and Snyder SH (1997) Urinary bladder-urethral sphincter dysfunction in mice with targeted disruption of neuronal nitric oxide synthase models idiopathic voiding disorders in humans. Nat Med 3:571-574.

Caremel R, Oger-Roussel S, Behr-Roussel D, Grise P, and Giuliano FA (2010) Nitric oxide/cyclic guanosine monophosphate signalling mediates an inhibitory action on sensory pathways of the micturition reflex in the rat. Eur Urol 58:616-625.

Cicek FA, Kandilci HB, and Turan B (2013) Role of ROCK upregulation in endothelial and smooth muscle vascular functions in diabetic rat aorta. Cardiovasc Diabetol 12:51.

Dimmeler S, Fleming I, Fisslthaler B, Hermann C, Busse R, and Zeiher AM (1999) Activation of nitric oxide synthase in endothelial cells by Akt-dependent phosphorylation. Nature 399:601-605.

Draganski A, Tar MT, Villegas G, Friedman JM, and Davies KP (2018) Topically applied curcumin-loaded nanoparticles treat erectile dysfunction in a rat model of type-2 diabetes. J Sex Med 15:645-653.

Förstermann U and Sessa WC (2012) Nitric oxide synthases: regulation and function. Eur Heart $J$ 33:829-837, 837a-837d.

Friedman AJ, Han G, Navati MS, Chacko M, Gunther L, Alfieri A, and Friedman JM (2008) Sustained release nitric oxide releasing nanoparticles: characterization of a novel delivery platform based on nitrite containing hydrogel/glass composites. Nitric Oxide 19:12-20.

Gallagher PJ, Herring BP, and Stull JT (1997) Myosin light chain kinases. J Muscle Res Cell Motil 18:1-16.

Grassie ME, Moffat LD, Walsh MP, and MacDonald JA (2011) The myosin phosphatase targeting protein (MYPT) family: a regulated mechanism for achieving substrate specificity of the catalytic subunit of protein phosphatase type $1 \delta$. Arch Biochem Biophys 510:147-159.

Han G, Tar M, Kuppam DS, Friedman A, Melman A, Friedman J, and Davies KP (2010) Nanoparticles as a novel delivery vehicle for therapeutics targeting erectile dysfunction. J Sex Med 7:224-233.

Huang PL, Dawson TM, Bredt DS, Snyder SH, and Fishman MC (1993) Targeted disruption of the neuronal nitric oxide synthase gene. Cell 75:1273-1286. 
Hurt KJ, Musicki B, Palese MA, Crone JK, Becker RE, Moriarity JL, Snyder SH, and Burnett AL (2002) Akt-dependent phosphorylation of endothelial nitric-oxide synthase mediates penile erection. Proc Natl Acad Sci USA 99:4061-4066.

Hurt KJ, Sezen SF, Lagoda GF, Musicki B, Rameau GA, Snyder SH, and Burnett AL (2012) Cyclic AMP-dependent phosphorylation of neuronal nitric oxide synthase mediates penile erection. Proc Natl Acad Sci USA 109:16624-16629.

Kamiyama Y, Muto S, Masuda H, Ide H, Ishizuka N, Saito K, and Horie S (2008) Inhibitory effects of nicorandil, a K ATP channel opener and a nitric oxide donor, on overactive bladder in animal models. BJU Int 101:360-365.

Karakus S, Anele UA, Silva FH, Musicki B, and Burnett AL (2019) Urinary dysfunction in transgenic sickle cell mice: model of idiopathic overactive bladder syndrome. Am J Physiol Renal Physiol 317:F540-F546.

Karakus S, Musicki B, La Favor JD, and Burnett AL (2017) cAMP-dependent posttranslational modification of neuronal nitric oxide synthase neuroprotects penile erection in rats. BJU Int 120:861-872.

Kizub IV, Pavlova OO, Johnson CD, Soloviev AI, and Zholos AV (2010) Rho kinase and protein kinase $\mathrm{C}$ involvement in vascular smooth muscle myofilament calcium sensitization in arteries from diabetic rats. $\mathrm{Br} J$ Pharmacol 159:1724-1731.

Laufs U and Liao JK (1998) Post-transcriptional regulation of endothelial nitric oxide synthase mRNA stability by Rho GTPase. J Biol Chem 273:24266-24271.

Lavelle JP, Meyers SA, Ruiz WG, Buffington CA, Zeidel ML, and Apodaca G (2000) Urothelial pathophysiological changes in feline interstitial cystitis: a human model. Am J Physiol Renal Physiol 278:F540-F553.

Leiria LO, Sollon C, Báu FR, Mónica FZ, D’Ancona CL, De Nucci G, Grant AD, Anhê GF, and Antunes E (2013) Insulin relaxes bladder via PI3K/AKT/eNOS pathway activation in mucosa: unfolded protein response-dependent insulin resistance as a cause of obesity-associated overactive bladder. J Physiol 591:2259-2273.

Meng E, Young JS, Cha TL, Sun GH, Yu DS, and Brading AF (2012) Neuronalderived nitric oxide modulates the activity of mouse detrusor smooth muscle. Neurourol Urodyn 31:572-578.

Ming XF, Viswambharan H, Barandier C, Ruffieux J, Kaibuchi K, Rusconi S, and Yang Z (2002) Rho GTPase/Rho kinase negatively regulates endothelial nitric oxide synthase phosphorylation through the inhibition of protein kinase B/Akt in human endothelial cells. Mol Cell Biol 22:8467-8477.

Mónica FZ, Bricola AA, Báu FR, Freitas LL, Teixeira SA, Muscará MN, Abdalla FM, Porto CS, De Nucci G, Zanesco A, et al. (2008) Long-term nitric oxide deficiency causes muscarinic supersensitivity and reduces beta(3)-adrenoceptor-mediated relaxation, causing rat detrusor overactivity. $\mathrm{Br} J$ Pharmacol 153:1659-1668.

Mondaini N, Ponchietti R, Muir GH, Montorsi F, Di Loro F, Lombardi G, and Rizzo M (2003) Sildenafil does not improve sexual function in men without erectile dysfunction but does reduce the postorgasmic refractory time. Int J Impot Res $\mathbf{1 5}$ $225-228$.

Moon A (2002) Influence of nitric oxide signalling pathways on pre-contracted human detrusor smooth muscle in vitro. BJU Int 89:942-949.

Morishita T, Tsutsui M, Shimokawa H, Sabanai K, Tasaki H, Suda O, Nakata S, Tanimoto A, Wang KY, Ueta Y, et al. (2005) Nephrogenic diabetes insipidus in mice lacking all nitric oxide synthase isoforms. Proc Natl Acad Sci USA 102 10616-10621.

Musicki B, Anele UA, Campbell JD, Karakus S, Shiva S, Silva FH, and Burnett AL (2019) Dysregulated NO/PDE5 signaling in the sickle cell mouse lower urinary tract: reversal by oral nitrate therapy. Life Sci 238:116922.

Musicki B, Champion HC, Becker RE, Liu T, Kramer MF, and Burnett AL (2005) Erection capability is potentiated by long-term sildenafil treatment: role of blood flow-induced endothelial nitric-oxide synthase phosphorylation. Mol Pharmacol 68: $226-232$.

Ozawa H, Chancellor MB, Jung SY, Yokoyama T, Fraser MO, Yu Y, de Groat WC and Yoshimura N (1999) Effect of intravesical nitric oxide therapy on cyclophosphamide-induced cystitis. J Urol 162:2211-2216.

Pandita RK, Mizusawa H, and Andersson KE (2000) Intravesical oxyhemoglobin initiates bladder overactivity in conscious, normal rats. $J$ Urol 164:545-550.

Parsons CL (2007) The role of the urinary epithelium in the pathogenesis of interstitial cystitis/prostatitis/urethritis. Urology 69 (4 Suppl):9-16.

Pászty C, Brion CM, Manci E, Witkowska HE, Stevens ME, Mohandas N, and Rubin EM (1997) Transgenic knockout mice with exclusively human sickle hemoglobin and sickle cell disease. Science 278:876-878.

Persson K, Pandita RK, Aszòdi A, Ahmad M, Pfeifer A, Fässler R, and Andersson KE (2000) Functional characteristics of urinary tract smooth muscles in mice lacking cGMP protein kinase type I. Am J Physiol Regul Integr Comp Physiol 279: R1112-R1120.

Portocarrero ML, Portocarrero ML, Sobral MM, Lyra I, Lordêlo P, and Barroso U Jr. (2012) Prevalence of enuresis and daytime urinary incontinence in children and adolescents with sickle cell disease. J Urol 187:1037-1040.

Powell LC, Szabo SM, Walker D, and Gooch K (2018) The economic burden of overactive bladder in the United States: a systematic literature review. Neurourol Urodyn 37:1241-1249.

Silva FH, Karakus S, Musicki B, Matsui H, Bivalacqua TJ, Dos Santos JL, Costa FF, and Burnett AL (2016) Beneficial effect of the nitric oxide donor compound 3-(1,3dioxoisoindolin-2-yl)benzyl nitrate on dysregulated phosphodiesterase 5, NADPH oxidase, and nitrosative stress in the sickle cell mouse penis: implication for priapism treatment. J Pharmacol Exp Ther 359:230-237.

Sriram K, Laughlin JG, Rangamani P, and Tartakovsky DM (2016) Shear-induced nitric oxide production by endothelial cells. Biophys $J$ 111:208-221.

Sutherland RS, Kogan BA, Piechota HJ, and Bredt DS (1997) Vesicourethral function in mice with genetic disruption of neuronal nitric oxide synthase. J Urol 157:1109-1116.

Tar M, Cabrales P, Navati M, Adler B, Nacharaju P, Friedman AJ, Friedman J, and Davies KP (2014) Topically applied NO-releasing nanoparticles can increase intracorporal pressure and elicit spontaneous erections in a rat model of radical prostatectomy. J Sex Med 11:2903-2914.

Teixeira CE, Jin L, Priviero FB, Ying Z, and Webb RC (2007) Comparative pharmacological analysis of Rho-kinase inhibitors and identification of molecular components of $\mathrm{Ca} 2+$ sensitization in the rat lower urinary tract. Biochem Pharmacol 74:647-658.

Wróbel A, Doboszewska U, Rechberger E, Rojek K, Serefko A, Poleszak E, SkalickaWoźniak K, Dudka J, and Wlaź P (2017) Rho kinase inhibition ameliorates cyclophosphamide-induced cystitis in rats. Naunyn Schmiedebergs Arch Pharmacol 390:613-619.

Yanai Y, Hashitani H, Hayase M, Sasaki S, Suzuki H, and Kohri K (2008) Role of nitric oxide/cyclic GMP pathway in regulating spontaneous excitations in detrusor smooth muscle of the Guinea-pig bladder. Neurourol Urodyn 27:446-453.

Address correspondence to: Dr. Serkan Karakus, The James Buchanan Brady Urological Institute, Johns Hopkins School of Medicine, 600 North Wolfe St., Marburg 414, Baltimore, MD 21287-2101. E-mail: skaraku2@ jhmi.edu 